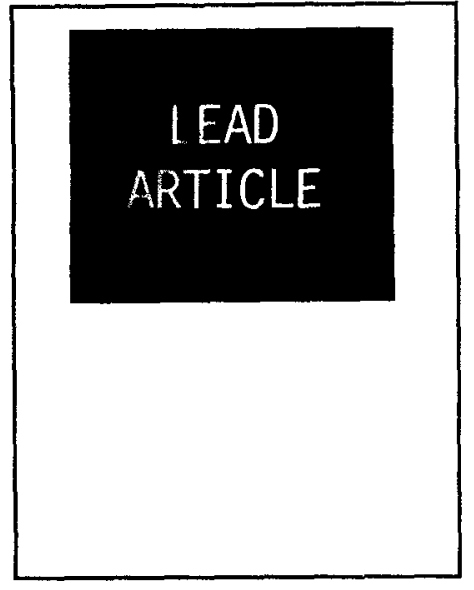

Abstract

Thirty Patients of Unsafe chronic suppurative otitis media were subjected to pre-operative CT scanning followed by surgical exploration of the middle ear and mastoid. and their scans were compared with the peroperative data. High resolution $C T$ scanning has been advocated for evaluation of unsafe chronic suppurative otitis media as it is capable of delineating detail required to detect Labyrinthine fistulae, Facial canal erosion, Sinus and Dural plate erosion and Ossicular integrity. Our results showed $C T$ scan to be highly sensitive for soft tissue density mass in the middle ear and mastoid. Dural plate exposure, Sinus plate erosion, Facial canal and Stapes integrity, moderately sensitive for Malleus and Incus integrity and least sensitive for Lateral canal fistulae. Both Axial and Coronal scans were done as many important structures are best seen in only one of these planes. The principal merit of $C T$ scan of the Tomporal bone lies in its inherent ability to depict pathology which is not clinically evident.

\section{Role of Computed}

\section{Tomography in Unsafe} Chronic Suppurative

\section{Otitis Media}

\section{Sandeep Berry Senior Resident, Dept. of}

ENT, Lok Nayak Hospital

S. C. Gandotra Consultant and Head, Dept. of

ENT, Safdarjans Hospital

N. C. Saxena : Consultant and Head Dept. of

Radiology, Safdarjang Hospital, New Delhi 100029

IT

Iigh resolution thin section computed tomography has widely replaced conventional techniques for temporal bone imaging. The most significant use of computed tomography lies in evaluation of cases of chronic suppurative otitis media. ( $O^{\prime}$ Reilly etal, 1991). High resolution CT of temporal bone has been implicated as a mode of diagnosing cholesteatoma (Mafee et al, 1988, Jackler et al, 1984), demonstrating ossicular chain (Schwartz, 1983), analysing labyrinthine fistulae (Bates et al, 1988) and detecting bony defects in facial canal (Johnson et al, 1983)

To formulate the above objective, 30 cases of unsafe chronic supurrative otitis media were subjected to computed tomography scanning of temporal bone prior to mastoind exploration. The pre-operative CT films were analysed by experienced Radiologist and answers pertaining to following questions were sought without the benefit of peroperative findings :

1. Presence of soft tissue mass in middle ear and mastoid.

2. Presence of bone erosion.

3. Integrity of sinus and dural plate.

4. Ossicular status.

5. Facial canal erosion.

6. Lateral canal fistulae.

The answers to above questions given by radiologist were then compared with peroperative findings and an attempt was made to evaluate the role computed tomography in unsafe CSOM :

\section{Methodology}

Computed tomography was performed on General electiric (GE) scanner using high resolution thin section (1-2 $\mathrm{mm}$ ) sections. Traditionally both coronal and axial sections were performed. Axial scans were taken at a plane 30 degrees above zero degree base line (orbito-meatal line). Coronal scans, on other hand, were done with the patient either prone with maximally extended or 


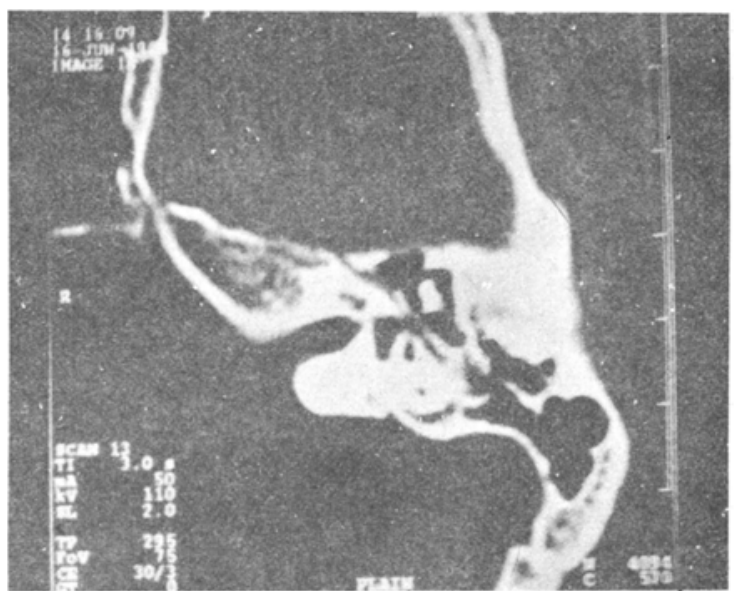

Fig: 1 Normal CT Temporal Bone (Axialcut) Showing Horizontal Portion of the Facial Nerve, Vestibule, Cochlea, Malleus, Incus, IncudoMalleal Joint and Internal Auditory Canal

supine and section being at an angle of 105 degree (Fig : 1, 2)

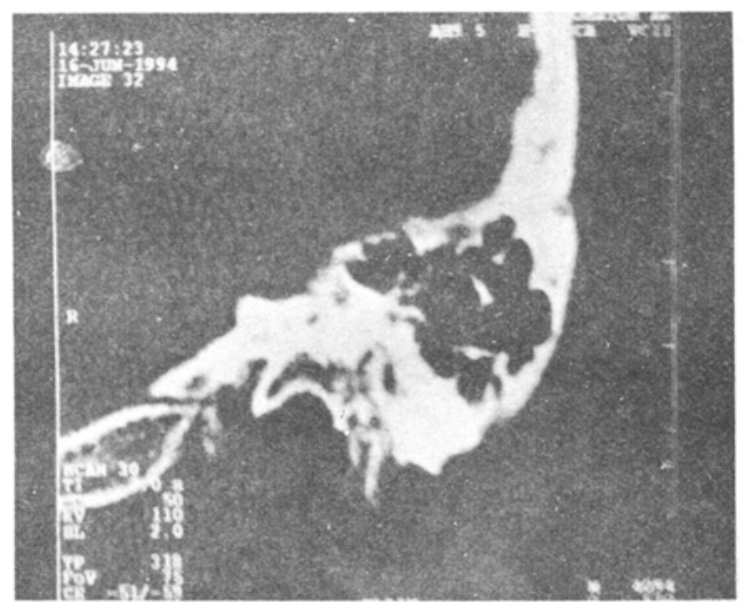

Fig: 2 Normal CT Temporal Bone (Coronal Cut) Showing vertical Portion of the Facial nerve, Mastoid Air cells and Dural Plate

\section{Discussion}

\section{- Soft Tissue Mass}

In present series, soft tissue masses were detected in each and every case in the middle ear and mastoid. However, the division between cholesteatoma and other soft tissue masses could not be made out preoperatively on CT. Peroperatively cholestecatoma was observed in 17 $(56.66 \%)$ cases, granulations alone without cholesteatoma in $11(36.66 \%)$ cases and polyp and purulent material along with granulations in $1(3.34 \%)$ case each respectively. Jackler et al (1984) found cholesteatoma to be present in $30 \%$ of cases where soft tissue mass along with bone erosion was present. Mafee etal (1988) stated that hallmarks of cholesteatoma on CT scan are a soft tissue mass in the attic and antrum along with smooth bony expansion, scalloping of the mastoid, erosion of lateral attic wall, erosion of anterior tympanic spine and erosion of ossicles.

\section{- Integrity of Dural and Sinus Plate}

As regarding integrity of sinus and dural plate on CT temporal bone, it was observed that in $9(30 \%)$ cases dura was exposed whereas erosion of sinus plate was seen in $6(20 \%)$ cases. However peroperatively, erosion of dural plate was seen in $7(23.34 \%)$ cases and that of sinus plate in $2(6.66 \%)$ cases. Thus, out of 9 cases showing dural plate erosion on $\mathrm{CT}$, only in $6(66.66 \%)$ cases it was confirmed peroperatively whereas in $3(33.34 \%$ ) cases, CT gave an erroneous impression of dural plate erosion (Fig3)) Similarly. out of 6 cases demonstrating sinus plate patholgy on $\mathrm{CT}$, only in $2(33.34 \%)$ it was confirmed and rest of $4(66.66 \%)$ cases gave a false imperession on CT O'Reilly etal (1991) stated that it is not possible to reliably show an erosion in the tegmen on axial cuts alone but even using coronal cuts, the effect of partial volume averaging gives a false impression of a defect. (fig.4) 


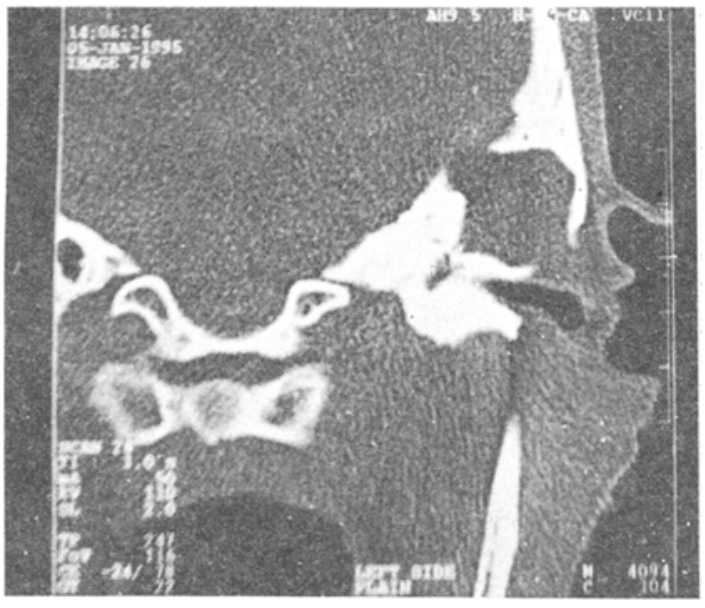

Fig : 3 Normal CT Temporal Bone (Coronal Cut) Showing Erosion of the Dural Plate by the Disease process which was also confirmed peropearatively

\section{- Lateral Canal Fistulae}

As stated by Sheehy et al (1977), Lateral canal fistulae occur in about $10 \%$ of cases of chronic suppurative otitis media. In present series, lateral canal was observed to be intact in $25(83.33 \%$ ) cases, erosion present in 1(3.33\%) and not properly seen in $4(13.34 \%)$ cases on CT. Peroperatively, lateral canal was intact in $27(90 \%)$ cases and eroded in $3(10 \%)$ cases. However, 1 case in which CT showed lateral canal fistulae peroperatively it was intact. Erosion seen in $3(10 \%)$ cases peroperatively was not delienated by $\mathrm{CT}$ scan in any of the cases. Also $4(13.34 \%)$ cases in which lateral canal was not properly visualised on CT, was found intact on exploration. It is important to realise that axial scans can give a false imprint of lateral canal fistulae (Mafee etal, 1988). On CT lateral canal can be seen in full length on axial scan in cross section coronally. On coronal scan, it often appears dehiscent on the medial wall of the antrum due to the volume averaging of the thin wall, in the presence of an soft tissue

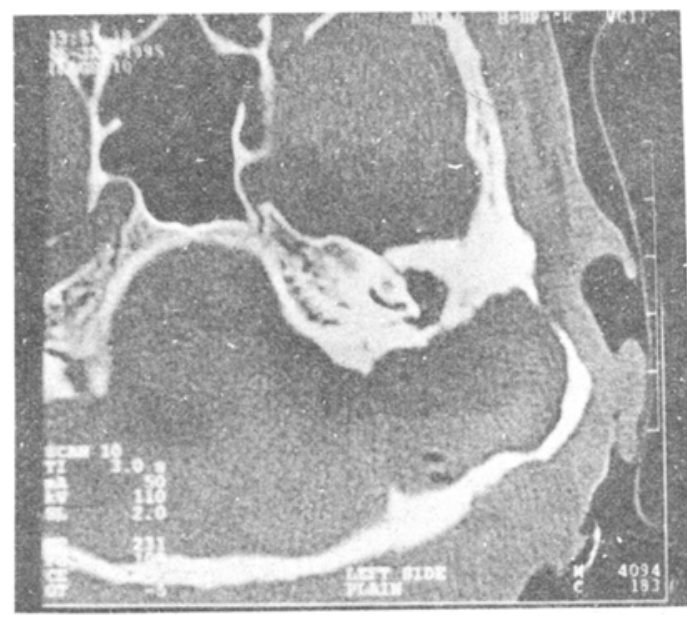

Fig : 4 Normal CT Temporal Bone (Axial Cut) Showing Erosion of the Sinus Plate by Cholesteatoma. However on mastoid Exploraion it Found to be Intact.

density mass, this may give the erroneous impression lateral canal fistulization. Thus for a proper information on the status of lateral canal, Axial scans (preferably tilted 30 degree to orbitc-meatal line) should be done. Jackler etal (1984) in their study observed a high incidence of false positives which was caused by sole reliance on coronal scans. (fig 5)

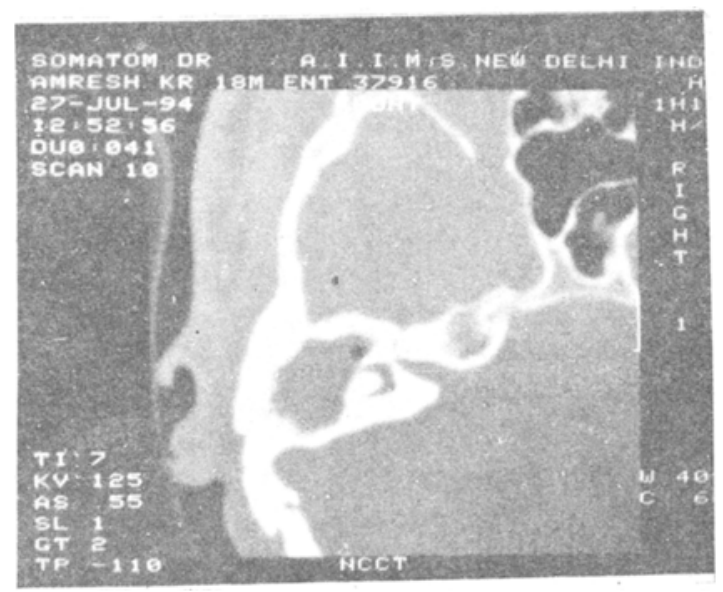

Fig: 5 CT Temporal Bone (Axial Cut) Showing a lateral Canal Fistula. Peroperatively the lateral canal was Found to be intact. 
Table No 1

Correlation between computed tomography and surgical findings :

\begin{tabular}{|lcc|}
\hline Feature & Peroperatively present & On CT \\
\hline Middle ear \& & 30 & 30 \\
Mastoid mass & & \\
Dural plate erosion & 07 & 09 \\
Sinus plate erosion & 02 & 06 \\
Ossicular necrosis & 21 & 00 \\
Lateral canal fistulae & 03 & 01 \\
Facial canal erosion & 05 & 00 \\
\hline
\end{tabular}

\section{- Facial canal}

In present study, facial canal was observed to be intact in $26(86.66 \%)$ cases and not clearly seen in $4(13.34 \%)$ cases on CT. However, peroperatively facial canal was intact in $25(83.34 \%)$ cases and erosion present in 5 $(16.66 \%)$ cases. Nevertheless out of the 4 cases in which facial canal was not visualized on $\mathrm{CT}$, it was found eroded in $3(75 \%)$ cases and intact in $1(25 \%)$ case on mastoid exploration. Facial nerve was found exposed in 2 cases peroperatively in which CT showed an intact facial canal. Mafee etal (1988) and Jackler etal (1984) stated that a soft tissue overlying the canal caused loss contrast gradient, obscuring a smali dehiscence. Jackler etal (1984) commented that the best technique for detecting erosion of bony canal is to view it with multiple crossectional slices. For this reason, coronal scan is prefered for the horizontal portion of facial nerve while axial scan is the view of the choice for the vertical portion. Mafee etal (1988) found agreement about facial nerve canal integrity in $41(85 \%)$ cases between radiographs and surgery. When the middle ear is disease free, thin lateral bony wall of fallopian canal is easy to visualize on CT scans against its black radioluscent background. But when pathologic soft tissue abuts the tympanic portion of the canal, it may be difficult to determine whether cholesteatoma has eroded the canal or merely lies adjacent to a congenital defect.

\section{- Ossicular Status}

It is relatively easy to visualize the bodies of malleus and incus on $\mathrm{CT}$ but this has little clinical value unless integrity of the whole ossicular chain is ascertained. The long process of Incus and Stapes suprastructure are most at risk in unsafe chronic suppurative otitis media but are also difficult to illustrate on CT. In present series, Malleus and Incus were seen to be intact in $18(60 \%)$ cases and absent in $12(40 \%)$ cases on CT whereas Stapes was intact in $1(3.33 \%)$ case and absent in 29 $(96.67 \%)$ cases. No definite comment could be made on the status of the ossicular chain necrosis on CT. Peroperatively Malleus was intact in $11(61.11 \%)$ cases, out of the 18 cases in which Malleus appeared intact on CT scan. In rest of the $7(38.89 \%)$ cases, CT gave a false impression of Malleus being intact. Peroperatively Malleus was necrosed in all of the above 7 cases. Out of the 12 cases in which malleus was absent on CT scan, in 9 (75\%) cases it was absent peroperatively and necrosed in $3(25 \%)$ cases. Thus, malleus was necrosed in $10(33.34 \%)$, intact 11 (36.66\%) and absent in $9(30 \%)$ cases peroperatively. Out of the 18 cases in which CT showed incus to be intact, only in $6(33.33 \%)$ cases it was confirmed peroperatively. In $12(66.67 \%)$ cases CT gave an faulty impression of incus being intact. Incus was found to be intact in $6(20 \%)$, necrosed in $8(26.66 \%)$ and absent in $16(53.34 \%)$ cases On mastoid exploration. 
Stapes was seen intact in $12(40 \%)$ cases peroperatively. However, $11(91.66 \%)$ of 12 cases of intact stapes were missed on CT scan. In out of 14 (48.27) of 29 cases in which stapes appeared absent on CT scan, it was found necrosed peroperatively in $3(21.42 \%)$ cases and intact in $11(78.58 \%)$ cases. Stapes was absent in $15(50 \%)$ cases peroperatively. Jackler etal (1984) stated that stapes was not consistently seen on CT. Phelps and Wright (1990) doubted that CT could reliably demonstrate the ossicular chain because of the combination of partial; volume averaging and tissue silhoueting.

\section{Conclusion}

The results of the present study showed $\mathrm{CT}$ temporal bone to be highly sensitive for dural plate exposure, sinus plate erosion, facial canal erosion and stapes integrity, moderately sensitive for malleus and incus sensitivity and least sensitive for lateral canal fistulae, when analysed statistically. However certain drawbacks of CT such as partial volume averaging do lead to false interpretation of the disease process. CT scan has a role in the evaluation of cases of unsafe chronic suppurative otitis media but must be interpreted with caution in view of its certain limitations.

1. Both axial and coronal scans should be done as important structures are best seen only on one of these planes.

2. CT occasionally gives an erroneous impression of lateral canal fistula, tegmen erosion and facial canal erosion due to partial volume averaging of the thin bone coverning of these structures with adjacent soft tissues.

3. Abnormal soft tissue associated with bone erosion on CT is highly correlated with cholesteatoma.

4. In present study, the findings of CT scan corroborated well with the peroperative findings

\section{Referenees}

1. Mafee, M. F., Levin, B. C., Applebaurn, E. L., Campos, M. and james, C. F. (1988) : Cholesteatoma of the middle ear and mastoid. A comparison of CT and operative findings. Otolaryngologic clinics of North America 24 (2) : 265-293.

2. O'Reilly, B. J., Chevretton, E. B., Wylie, I., Thakkar, C. Butler, P, Sathanathan, N. Morrison, G. A. and Kenyon G.S. (1991) : The value of CT scanning in chronic suppurative otitis media. The journal of laryngology and otology 105 : 990-994.

3. Jackler, R. K., Dillon, W. P. and Schindler, R. A. (1984) : Computed tomography in suppurative ear disease : A correlation of surgical and radiographic findings. Laryngoscope 94 : 746-752.

4. Bates, G.J., O'Donoghue, G. M., Anslow, P. and Houlding. T. (1988) : Can CT detect labyrinthine fistulae preoperatively? Acta otolaryngologica $106: 40-45$.

5. Johnson, D. W., Voorhees, R. L., Lufkin, R. B., Hanafee, W. and Canalis, R. (1983) : Cholesteatomas of the temporal bone: Role of computed tomography. Radiology $148: 733-737$.

6. Phelps, P. D. and Wright, A. (1990) : Imaging cholesteatoma. Clinical radiology. $41: 156-162$.

7. Sheehy, J. L., Brackmann, D. E., Graham, M. D. (1977) : Complications of cholesteatomas : a report of 1024 cases. In First International conference on cholesteatoma (McCabe, B. F., Sade, J., Abramson, M. Eds), Aesculapius Press, Birmingham, Alabama. : 420-429.

8. Swartz, J. D. (1983) : High resolution computed tomography of middle ear and mastoid. Radiology $148: 449-454$. 